\title{
A NOVEL MUTATION IN A NEWBORN BABY LEADING TO GLYCOGEN STORAGE DISEASE TYPE IA
}

\author{
Dorum $\mathrm{S}^{1, *}$, Gorukmez $\mathrm{O}^{2}$ \\ *Corresponding Author: Sevil Dorum, M.D., Department of Pediatrics, Division of Metabolism, \\ Bursa Yüksek İhtisas Training and Research Hospital, Emniyet Street 35, 16310, Yıldırım, Bursa, Turkey. \\ Tel.: +90+505-258-3766. Fax: +90-224-294-4000. E-mail: sevildorum@gmail.com
}

\begin{abstract}
Glycogen storage disease type Ia (GSD1A) is caused by mutations in the G6PC gene. The G6PC gene was first cloned in 1993. Since then, many different mutations have been identified leading to this disease. Hepatomegaly is one of the important clinical manifestations of the disease. A 23-day-old girl was admitted to the hospital due to respiratory distress. Her physical examination was normal except for tachypnea. She had hypoglycemia, lactic academia, hyperlipidemia and hyperuricemia. With these clinical findings, GSD1A was considered in the patient and the diagnosis was genetically confirmed. By direct sequencing of the G6PC gene, we identified a novel homozygous variation (c.137T $>$ G/p.Leu46Arg) in the patient and the healthy mother and father were heterozygotes for the variant. Here we present a case with a novel homozygous missense mutation c.137T $>$ G/p.Leu46Arg in the G6PC gene leading to GSD1A clinical findings except early hepatomegaly. These findings expand the spectrum of causative mutations, and clinical findings in GSD1A.

Keywords: Glycogen storage disease type Ia (GSD1A); Novel mutation; von Gierke disease.
\end{abstract}

\section{INTRODUCTION}

Glycogen storage disease type Ia (GSD1A; MIM: 232200 ) is an autosomal recessive disorder that is caused by deficient glucose-6-phosphatase (G6Pase) activity [1]. Hypoglycemia, lactatemia, hyperuricemia, hyperlipid-

\footnotetext{
${ }^{1}$ Department of Pediatrics, Division of Metabolism, Bursa Yuksek Ihtisas Training and Research Hospital, Bursa, Turkey

${ }^{2}$ Department of Genetics, Bursa Yuksek Ihtisas Training and Research Hospital, Bursa, Turkey
}

emia, and marked hepatomegaly are the leading features of this inborn error of metabolism [2]. Up to now, over 100 mutations have been identified in the G6PC gene [3]. Here we present a case with a novel homozygous missense mutation c. $137 \mathrm{~T}>\mathrm{G} / \mathrm{p}$.Leu46Arg in the G6PC gene leading to GSD1A clinical findings.

\section{CASE REPORT}

A 23-day-old girl was admitted to the emergency room because of respiratory distress. On physical examination, there was no abnormality except tachypnea, and there was no hepatomegaly. She had hypoglycemia $(32.0 \mathrm{mg} /$ $\mathrm{dL})$, lactatemia $(8.0 \mathrm{mmol} / \mathrm{L})$ and hyperuricemia $(9.2 \mathrm{mg} /$ dL). Other routine blood tests, urinalysis, and chest X-ray were normal. She was the first child of consanguineous parents. She was born at 38 weeks' gestation and weighed $2300 \mathrm{~g}$. Blood samples were obtained for metabolic tests. After hospitalization, hypertriglyceridemia $(569.0 \mathrm{mg} /$ $\mathrm{dL}$ ) was determined. The size of the liver was normal on the ultra-sonographic (USG) examination. Metabolic tests [tandem-mass spectrometry (MS), blood amino acid chromatography, urine organic acids] determined normally. With these findings, the patient was thought to carry GSD1A disease. Her daily diet was planned for $65.0 \%$ of total energy intake from carbohydrates, $15.0 \%$ from protein and the remainder from fat (with high linoleic acid content). Informed written consent was obtained from parents.

To confirm the diagnosis, mutational analysis of the G6PC gene was performed by direct DNA sequencing. Genomic DNA was isolated from peripheral blood of the proband and her family using a DNA isolation kit (RTA Laboratories, Kocaeli, Turkey). To identify any mutations the proband might be carrying, exons of the G6PC gene including exon/intron flanking regions, were amplified 
by polymerase chain reaction (PCR) with specific primers that were designed using Primer 3 software (http:// ihg.gsf.de/ihg/ Exon Primer.html) and H Taq polymerase (Zeydanlı, Ankara, Turkey). Standard PCR conditions with 35 cycles were used and performed on a 9700 Thermal Cycler (Applied Biosystems, Foster City, CA, USA). The amplified products were purified by using Zymo Research Sequencing Clean-up Kit (Epigenetic Companies, Irvine, CA, USA). Cycle sequencing was performed with the BigDye Terminator v3.1 Cycle Sequencing Kit (Applied Biosystems) on an Applied Biosystems ${ }^{\circledR} 3130 \mathrm{Ge}$ netic Analyzer. The sequence data were analyzed using sequencing analysis v5.3.1 software program (Applied Biosystems) and compared to the reference sequence (GenBank Accession Nos. NG_011808.1, NM_000151.3, NP_000142.2).

Lactatemia and hypertriglyceridemia have continued in the follow-up examinations. At 9 months of age, USG revealed mild hepatomegaly (craniocaudal length $=100$ $\mathrm{mm}$ ) for the first time, but there was no hepatomegaly on physical examination. Liver functions were normal.

\section{DISCUSSION}

Glycogen storage disease type Ia is a rare disease that primarily affects the kidneys and liver. There is an excessive accumulation of glycogen in the liver and kidneys due to G6Pase enzyme deficiency [2]. Patients with GSD1A have various clinical manifestations according to the patient's age, including fasting hypoglycemia, hepatomegaly, hyperlipidemia, lactic acidemia, hyperuricemia, poor growth and short stature $[1,2]$. In the neonatal period, patients may present with symptoms of lactic acidosis and hypoglycemia [4,5]. Our patient presented with hypoglycemia and lactic acidemia in the neonatal period. Glycogen storage disease type Ia was considered in our patient with other clinical findings and the diagnosis was genetically confirmed. By direct sequencing of the G6PC gene, we identified a novel homozygous variation (c.137T $>\mathrm{G} / \mathrm{p}$. Leu 46Arg in exon1) in the patient and the healthy mother and father were heterozygotes for the variant (Figure 1). This variant has not been previously reported in the Human Gene Mutation Database (HGMD; http://www. hgmd.cf.ac. uk/ac/ index.php) and in population studies (ExAC: Exome Aggregation Consortium and $1000 \mathrm{Ge}-$ nomes Project; http://exac. broadinstitute. org/). In silico analysis program (VarSome; DANN Score: 0.9967; https:// varsome.com/) showed that this change could be the cause of the disease.

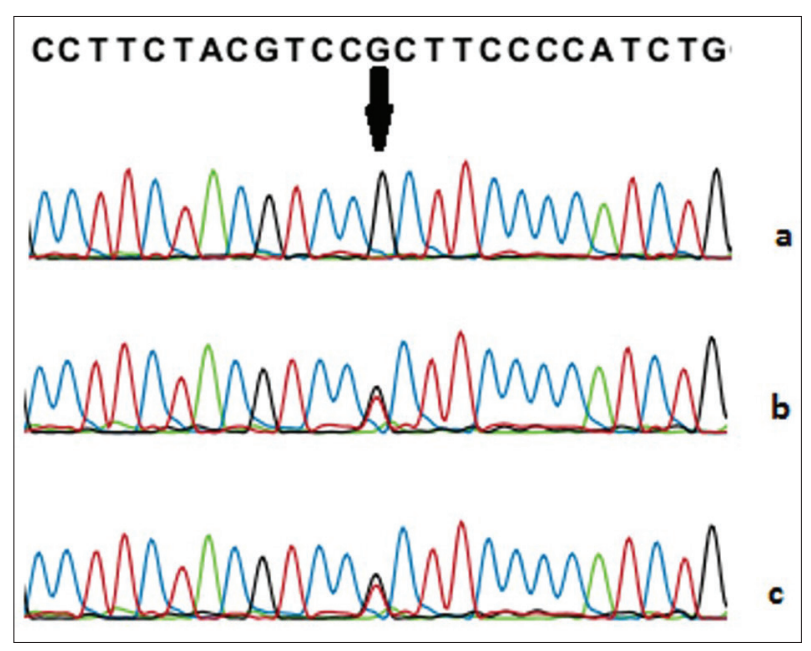

Figure 1. Sequencing analysis of the $G 6 P C$ gene c. $137 \mathrm{~T}>\mathrm{G}$ mutation. (a) The arrow shows the site of the homozygous c.137T $>\mathrm{G}$ mutation in the G6PC gene of the patient. (b) Heterozygous mutation carrier (mother). (c) Heterozygous mutation carrier (father).

Hepatomegaly is one of the main findings of GSD1A and it is seen in all patients but proper diagnosis can be difficult in infants with GSD1A who do not have not severe hepatomegaly. Infants who have not been diagnosed before are presenting with hepatomegaly at 3 to 6 months of age [2]. In these patients, hepatomegaly occurs due to glycogen and fat storage [7].

However, hepatomegaly was not detected either in the examination or in the USG in the follow-up of our patient during the first 9 months. First, at the end of the 9th month, USG examination revealed mild hepatomegaly and an increase of liver echogenicity.

In these patients, liver functions are normal except for glucose homeostasis, cirrhosis is not expected. Adenoma may develop in the second decade of life. Liver functions were normal in our patient. Dietary treatment improves the quality of life of the patients and may prevent complications [6]. Our patient's diet was regulated according to the European Study on Glycogen Storage Disease Type I recommendations [7].

We present a patient with GSD1A and a novel mutation in the G6PC gene. Our findings have expanded the spectrum of causative mutations, and clinical findings in GSD1A. This novel mutation, which was not previously described, appears to be a mutation associated with milder hepatomegaly.

Declaration of Interest. The authors report no conflicts of interest. The authors alone are responsible for the content and writing of this article. 


\section{REFERENCES}

1. Kishnani PS, Austin SL, Abdenur JE, Arn P, Bali DS, Boney A, et al. Diagnosis and management of glycogen storage disease type I: A practice guideline of the American College of Medical Genetics and Genomics. Genet Med. 2014; 16(11): e1.

2. Dambska M, Labrador EB, Kuo CL, Weinstein DA. Prevention of complications in glycogen storage disease type Ia with optimization of metabolic control. Pediatr Diabetes. 2017; 18(5): 327-331.

3. Sun A. Glucose-6-phosphatase deficiency (glycogen storage disease I, von Gierke disease). Hahn S, Editor. Waltham, MA, USA: UpToDate Inc. (https://www. upto date.com; accessed February 16, 2018.

4. Aydemir Y, Gurakan F, Saltik Temizel IN, Demir H, Karli Oguz K, et al. Evaluation of central nervous system in patients with glycogen storage disease type 1a. Turkish J Pediatr. 2016; 58(1): 12-18.
5. Hufton BR, Wharton BA. Glycogen storage disease (type 1) presenting in the neonatal period. Arch Dis Child. 1982; 57(4): 309-319.

6. Eminoğlu FT, Tümer L, Okur I, Ezgü FS, Hasanoğlu A. Clinical course and outcome of glycogen-storage disease type 1a and type 1b. Turk Arch Ped. 2013; 48(2): 117-122.

7. Rake JP, Visser G, Labrune P, Leonard JV, Ullrich K, Smit GPK. Guidelines for management of glycogen storage disease type I - European Study on Glycogen Storage Disease Type I (ESGSD I). Eur J Pediatr. 2002; 161(Suppl 1): 112-119. 